\title{
Influence of beam-coupling on photorefractive parametric oscillation in a dc-field-biased $\mathrm{Bi}_{12} \mathrm{SiO}_{20}$ crystal
}

\author{
Henrik C. Pedersen and D. J. Webb \\ Applied Optics Group, School of Physical Sciences, University of Kent, Canterbury, Kent CT2 7NR, UK \\ Per M. Johansen \\ Optics and Fluid Dynamics Department, Risb National Laboratory, DK-4000 Roskilde, Denmark
}

Received January 15, 1998; revised manuscript received April 13, 1998

\begin{abstract}
The influence of beam coupling on photorefractive parametric oscillation generated in a $\mathrm{Bi}_{12} \mathrm{SiO}_{20}$ crystal is investigated experimentally by comparing two configurations with and without the presence of beam coupling. It is shown that beam coupling has a great influence; for example, the transversal split of the $\mathbf{K} / 2$ subharmonic grating is seen only in the beam-coupling geometry. A case that resembles $\mathbf{K} / 4$ subharmonic generation can, however, still be found in the absence of beam coupling. (C) 1998 Optical Society of America [S0740-3224(98)01409-X]
\end{abstract}

OCIS codes: $160.5320,050.2770$.

\section{INTRODUCTION}

The first example of photorefractive parametric oscillation was discovered in 1989 by Mallick et al. ${ }^{1}$ The effect was observed during the performance of a so-called running grating experiment in a crystal of $\mathrm{Bi}_{12} \mathrm{SiO}_{20}$ (BSO) in which an electric-field-biased crystal is illuminated by a moving light interference pattern with grating vector $\mathbf{K}$ and frequency $\Omega$. At $\Omega=\omega_{\mathbf{K}}$ (the resonance frequency of the space-charge wave with wave vector $\mathbf{K})^{2}$ resonant excitation of a fundamental running grating, with wave vector $\mathbf{K}$ and frequency $\Omega$, takes place. ${ }^{3}$ However, when increasing $\Omega$ beyond $\omega_{\mathbf{K}}$, Mallick et al. ${ }^{1}$ found that additional gratings with grating vectors $\mathbf{K} / 2, \mathbf{K} / 3$, and $\mathbf{K} / 4$, respectively, were generated. Consequently, the effect was referred to as subharmonic generation, as the additional grating vectors assumed integer fractions of the fundamental grating vector $\mathbf{K}$. However, later on the term subharmonic generation was expanded to photorefractive parametric oscillation $(\mathrm{PPO})^{4}$ to cover the effects of broadening and splitting ${ }^{5-8}$ of the subharmonic gratings. With the observation of broadening, the question was raised of whether subharmonic generation is due to single gratings or rather to continua of gratings with grating vectors near to the subharmonic ones. This question will be explored further in the present paper.

In addition to the dc case, in which the crystal is biased by a dc electric field, the ac variant was discovered in which the electric biasing is performed with an alternating field instead of a dc field and the moving light pattern is replaced with a stationary one. ${ }^{9}$ In this case, too, $\mathbf{K} / 2$, $\mathbf{K} / 3$, and $\mathbf{K} / 4$ subharmonics were observed.

In the early days of theoretical modeling there was an intense discussion as to whether PPO was due to (i) beam coupling, in which light scattered from self-generated parasitic noise gratings is amplified in preferential direc- tions, or to (ii) inherent nonlinearities in the response of the material itself, without the influence of beam coupling. In fact, it could be both: beam coupling, because in the early experiments beam coupling was indeed possible and had previously been proven to cause beam fanning; material nonlinearities, because the intensity modulation used in the experiments was so large that nonlinear material effects could easily be the cause. The breakthrough in the discussion came in 1993, when McClelland et al. ${ }^{10}$ proved experimentally that the simplest case of PPO, namely $\mathbf{K} / 2$ subharmonic generation, could be generated in a setup in which beam coupling was not possible. Since then there has been a general consensus that PPO is a material effect, and thus the vast majority of theoretical models rely on this idea and disregard beam coupling. ${ }^{2,11-13}$ However, today still the majority of experiments on PPO have been performed in setups in which beam coupling is possible, ${ }^{1,4-9,14-16}$ and, importantly, little has been done to investigate the differences between experiments performed in the presence of beam coupling and others performed in its absence. Such investigations are nevertheless extremely important in view of the fact that there are still experimental features of PPO that the material-based theories fail to explain, such as why generation of the subharmonic $\mathbf{K} / 2, \mathbf{K} / 3$, and $\mathbf{K} / 4$ gratings tends to dominate over that of other gratings. Could it be that this feature is due to beam coupling?

This exact question was raised and investigated just recently in the ac case, ${ }^{17}$ and the results were striking. It appeared that by use of the exact same crystal in two different geometries (with and without beam coupling), $\mathbf{K} / 2$, $\mathbf{K} / 3$, and $\mathbf{K} / 4$ subharmonics were observed in the beamcoupling geometry but were absent in the geometry without beam coupling. In the latter case only a broad con- 
tinuum of gratings was seen without any division into packages of $\mathbf{K} / 2, \mathbf{K} / 3$, and $\mathbf{K} / 4$ gratings. So in the ac case it was concluded that the $\mathbf{K} / 2, \mathbf{K} / 3$, and $\mathbf{K} / 4$ division is due to beam coupling. A similar investigation would certainly be interesting for the dc case, as this case is the one that has attracted the most attention. Such an investigation is the aim of this paper. Another point of interest for the experimentalist is that the theoretical modeling of PPO seems to have entered a new era. Once concerned mainly with the thresholds of the instabilities that lead to PPO, ${ }^{2,11-13}$ the theorists have recently started describing the steady states of the process, revealing surprising features. ${ }^{18,19}$ For example, it has been proven that the simplest and most common PPO process, $\mathbf{K} / 2$ subharmonic generation, is in fact not stable in the steady state-despite the fact that $\mathbf{K} / 2$ subharmonic generation has the lowest threshold and thereby the largest increment (growth rate) of any PPO process. In other words, there might be a big difference between the transient growth of a PPO process and its final state; some gratings might be present only transiently and then vanish in steady state, or, conversely, some might not be present at the beginning of the growth but might turn up later as part of a stabilization process. Until now, the experimental reports on PPO have concentrated on describing the steady state, so with the newly discovered theoretical results in mind there is obviously a great need to perform transient investigations to verify whether there really are differences between the transient and steady states. The second aim of this paper is to initiate these investigations.

The paper is composed as follows. In Section 2 we report on PPO experiments performed in the traditional configuration in which beam coupling is possible. Only the steady state is considered here. Then, in Section 3, virtually the same experiment is performed but in a configuration without beam coupling. Moreover, the transient evolution of the PPO process is investigated in this configuration. All results are then discussed as a whole in Section 4.

\section{EXPERIMENTS IN THE BEAM- COUPLING GEOMETRY}

The experiment reported on in this section is practically identical to the one performed in Refs. 7 and 8. The reason for repeating it is that it is of great importance to perform two sets of experiments (with and without beam coupling) in the same crystal and under the same conditions in order to have a proper standard of reference. The experimental setup with the beam-coupling geometry is shown in Fig. 1. An expanded, linearly polarized laser beam from a frequency doubled ND:YAG laser is split in two and, by means of the three mirrors $M_{1-3}$, is sent toward the (110) face of a BSO crystal. The crystal dimensions are $13 \mathrm{~mm} \times 13 \mathrm{~mm} \times 6 \mathrm{~mm}$ along the $\langle 001\rangle,\langle 110\rangle$, and $\langle 1 \overline{1} 0\rangle$ crystallographic directions. The two beams, referred to as recording beams, form an interference pattern with an average intensity of $9.1 \mathrm{~mW} \mathrm{~cm}^{-2}$ and a fringe spacing $\Lambda=13 \mu \mathrm{m}$. The beam intensity ratio is $1: 1.9$, which gives an intensity modulation of 0.95 . The mirror $\mathrm{M}_{1}$ is mounted on a piezo stack to which a sawtooth voltage is applied ( $\Sigma$ in position 1 in Fig. 1 ). In this manner the frequency of the reflected recording beam is detuned by $\Omega$, and, consequently, the interference pattern moves uniformly in a direction perpendicular to the light interference fringes, i.e., along the $\langle 1 \overline{1} 0\rangle$ crystallographic direction. The interference pattern is thus characterized

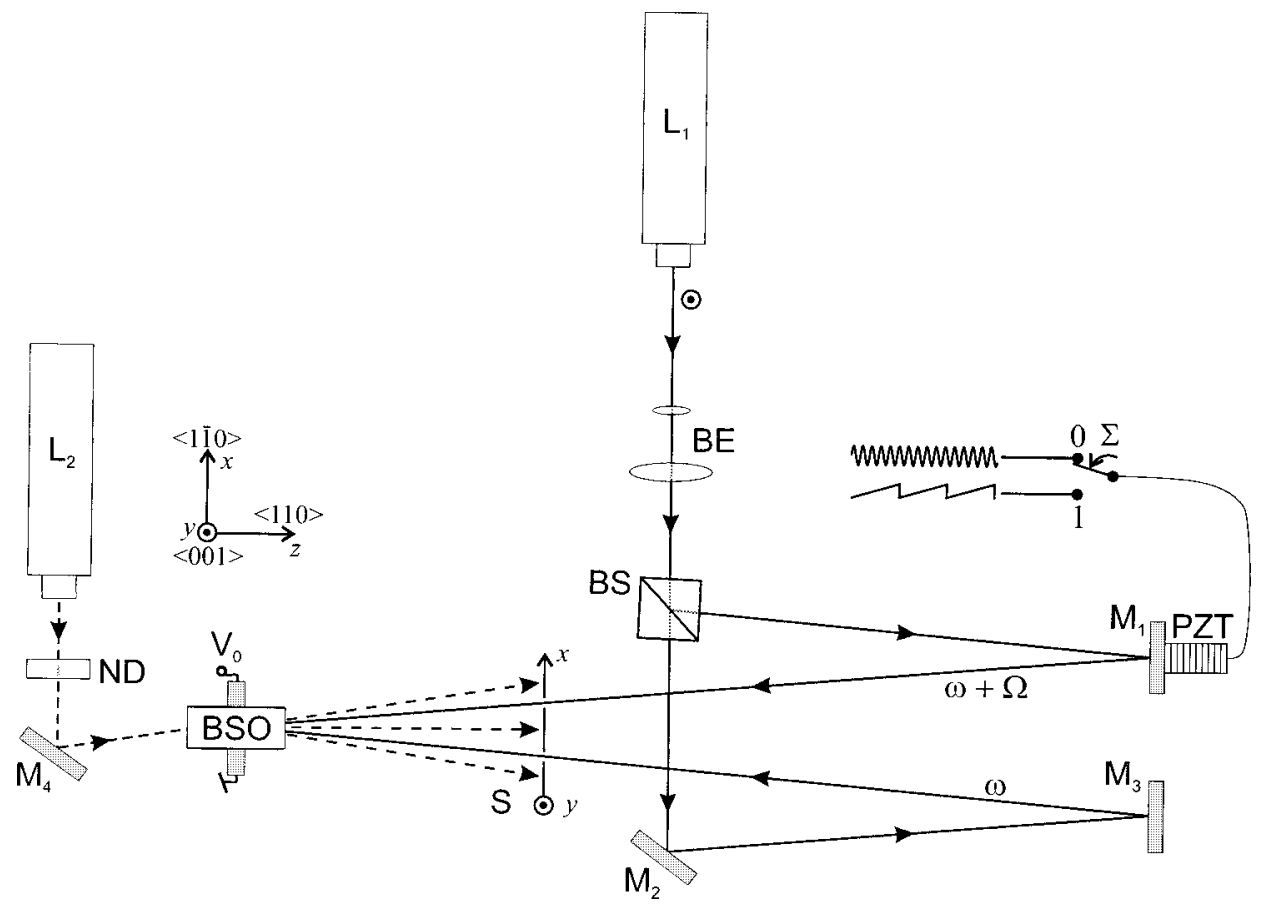

Fig. 1. Experimental setup used for beam-coupling experiments. $L_{1}$, diode pumped, frequency doubled ND:YAG laser emitting light at $\lambda=532 \mathrm{~nm} ; \mathrm{L}_{2}$, He-Ne laser emitting light at $\lambda=633 \mathrm{~nm}$; BE, beam expander; BS, beam splitter; ND, neutral-density filter; $\Sigma$, electrical switch; PZT, piezo stack; S, screen; and M's, mirrors. $\omega$ is the ND:YAG laser frequency and $\Omega$ is a frequency detuning induced by the moving piezo mirror. 


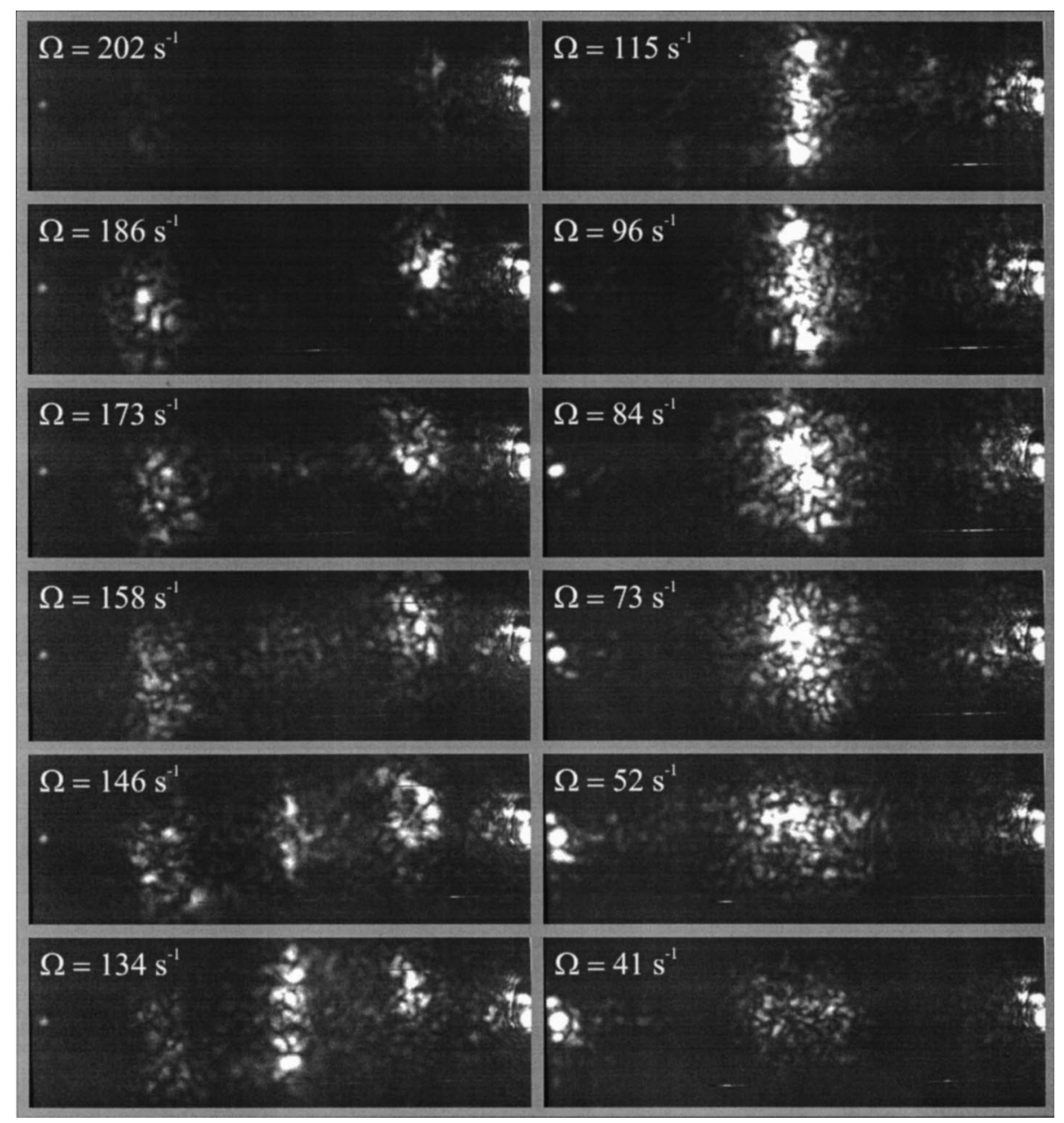

Fig. 2. Diffraction patterns observed at the screen in the beam-coupling geometry. The spot on the left is due to diffraction in the fundamental grating; the one on the right is due to the directly transmitted readout beam. The central part of the latter spot has been damped to avoid overflow of the CCD camera; the broadened pattern around this spot is due to simple scattering in the crystal. The patterns in between are due to PPO. The horizontal and vertical directions follow the $x$ and $y$ axes, respectively, shown in Fig. 1.

by a grating vector $\mathbf{K}=\hat{x} 2 \pi / \Lambda$, where $\hat{x}$ is a unit vector along $\langle 1 \overline{1} 0\rangle$ and by a temporal frequency $\Omega$ governed by the slope of the sawtooth voltage. Apart from that a dc voltage $\mathrm{V}_{0}$ of $5 \mathrm{kV}$ is applied to the crystal by means of two silver electrodes painted on the crystal's two (110) faces. Thus an electric field of $8.3 \mathrm{kV} \mathrm{cm}^{-1}$ is induced along the $\langle 1 \overline{1} 0\rangle$ direction.

The geometry in this setup is called the beam-coupling geometry because the recording beams are capable of diffracting from the grating they record themselves and are thereby capable of exchanging energy. Whether beam coupling is possible is determined by the electro-optic tensor of the crystal. ${ }^{20}$

The recorded holograms are read out with a $\mathrm{He}-\mathrm{Ne}$ laser at a read-out angle that is approximately half the Bragg angle of the fundamental grating. By means of a neutral-density filter the power of the readout beam is reduced to $1 \mathrm{~mW}$ to ensure that the readout beam does not participate in the recording or erasure of holograms (socalled nondestructive readout). This is easily checked by quickly turning on the readout beam and seeing whether the observed diffraction pattern appears instantaneously. Instantaneous appearance implies a nondestructive read- out. The resulting diffraction patterns are projected onto screen $\mathrm{S}$ shown in Fig. 1 and then subsequently recorded by a CCD camera.

By decreasing the frequency detuning $\Omega$ stepwise through the interval $202-41 \mathrm{~s}^{-1}$, the diffraction patterns shown in Fig. 2 were observed. After each step in $\Omega$ we waited long enough for the patterns to reach steady state or rather as close to steady state as possible, because the patterns do keep changing shape to a certain extent. It was possible, though, to obtain pictures that represent each of the cases quite well. It is seen in Fig. 2 that as $\Omega$ was changed various cases of PPO appeared. For high values of $\Omega$, two spots centered roughly at $\mathbf{K} / 4$ and $3 \mathbf{K} / 4$ appeared. The intensity of these spots increased as $\Omega$ was decreased, reaching a maximum at approximately $\Omega=150 \mathrm{~s}^{-1}$. At the same time a new spot centered at $\mathbf{K} / 2$ appeared. When $\Omega$ was decreased further, the $\mathbf{K} / 4$ and $3 \mathbf{K} / 4$ spots gradually vanished whereas the $\mathbf{K} / 2$ spot tended to split up in a direction transversal to $\mathbf{K}$. This spit was most pronounced around $\Omega=115 \mathrm{~s}^{-1}$. When $\Omega$ was lowered even more, the transversal split pattern turned into a more-or-less broad spot around $\mathbf{K} / 2$. The dependence of the different pattern shapes on $\Omega$ re- 
sembles very much that observed in Refs. 7 and 8 . It should be mentioned that the transversal split of the $\mathbf{K} / 2$ spot could be made much more pronounced by increasing the applied electric field.

\section{EXPERIMENTS WITHOUT BEAM COUPLING}

In the next experiment we switch from beam-coupling geometry to the geometry introduced by McClelland et al., ${ }^{10}$ in which beam coupling cannot take place. The geometry is illustrated in Fig. 3, showing only the central part of the setup. The new geometry is obtained by simply tilting the two mirrors $\mathrm{M}_{1,3}$ (see Fig. 1) so that the two recording beams exactly clear the top of the crystal, thus impinging on a new mirror $\mathrm{M}_{5}$ situated above the crystal (Fig. 3) and illuminating the top (001) crystal face. In this way an interference pattern, with $\mathbf{K}$ and $\Omega$ identical to those in the previous geometry, is formed. However, propagating virtually along the $\langle 001\rangle$ crystal axis, the two recording beams here are unable to diffract from the anisotropic grating they record. Hence beam coupling is not possible. ${ }^{20}$

A very important parameter in the theory of parametric oscillation is the detuning parameter $\epsilon=\omega_{\mathbf{K}} / \Omega$, where $\omega_{\mathbf{K}}$ is the linear resonance frequency (see, for example, Ref. 2). In the so-called linear-in-modulation approximation, i.e., for sufficiently small values of the intensity modulation $m$, the linear resonance frequency is easily measured as the value of $\Omega$ at which maximum diffraction efficiency is obtained. If $m$ is too close to unity, higher-order gratings may be present, thus causing a nonlinear shift of the resonance frequency. ${ }^{21,22}$ To re- duce $m$ we therefore attenuated the weakest recording beam by $10^{3}$, reducing $m$ to 0.046 , and, at the same time, the readout angle was provisionally set to Bragg match the fundamental grating. In this setup we measured a resonance frequency of $15.1 \mathrm{~s}^{-1}$. As the resonance frequency scales linearly with the dc intensity $I_{0}$, the resonance frequency at full illumination, that is, in the absence of the attenuation, scales up to $\omega_{\mathbf{K}}=23 \mathrm{~s}^{-1}$.

\section{A. Steady State}

In the first series of experiments we concentrate on the steady state, as in Section 2. As above, we scanned the frequency detuning $\Omega$ through an interval that is sufficient to bring out all characteristic cases of PPO while the readout angle was reset to half the fundamental Bragg

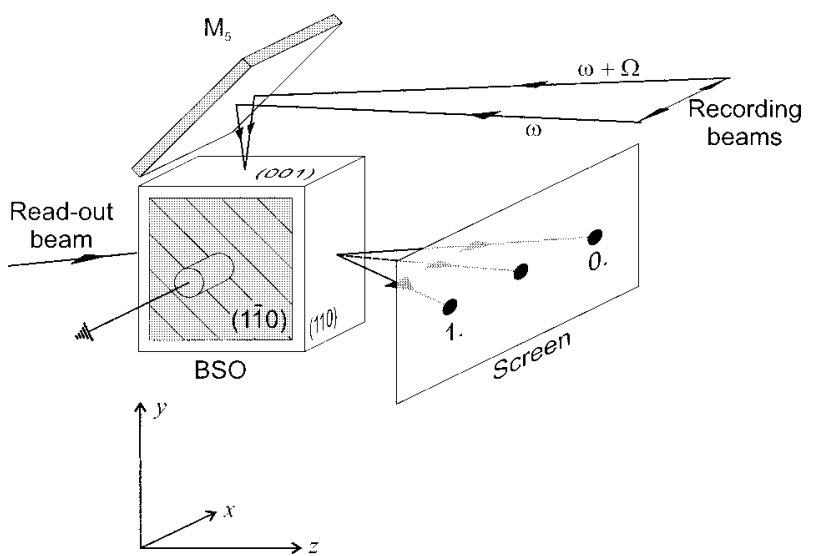

Fig. 3. Central part of the configuration without beam coupling in which the recording beams have been redirected to impinge the crystal's upper (001) face.

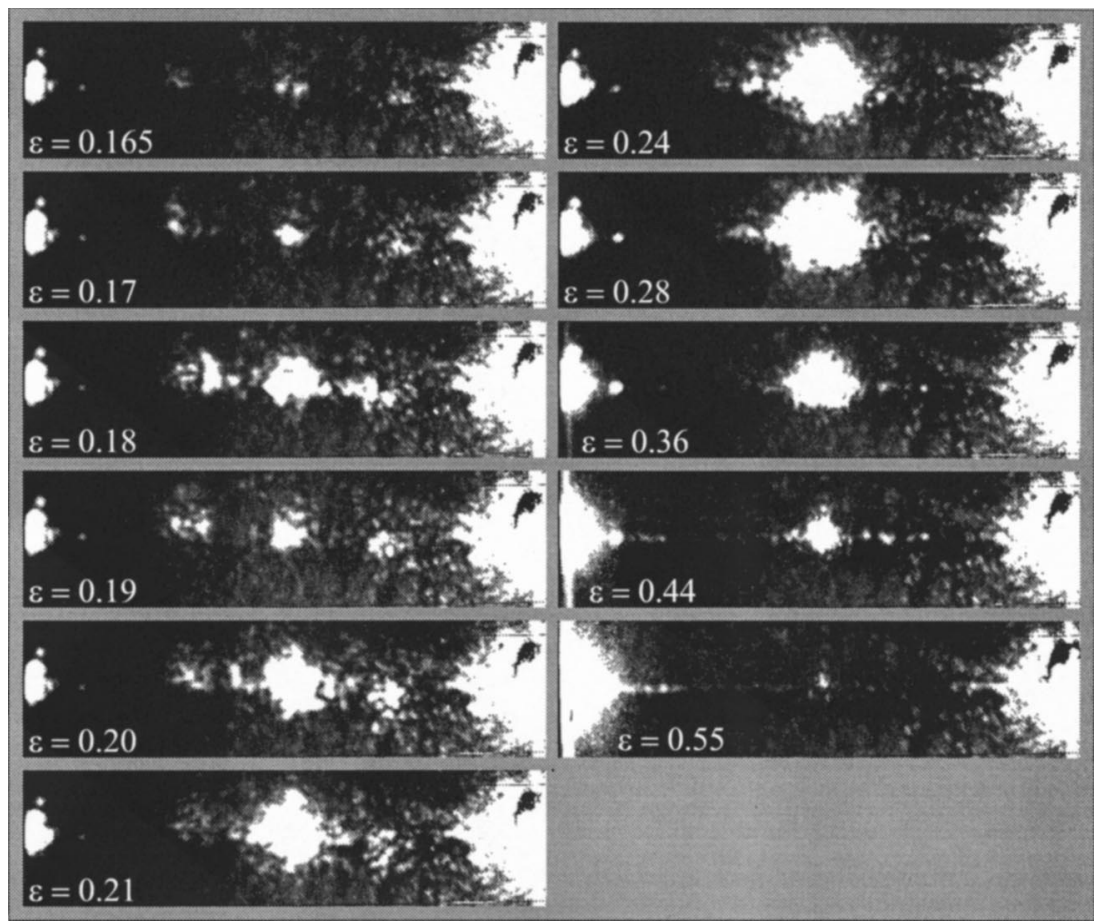

Fig. 4. Diffraction patterns in steady state obtained in the geometry in which beam coupling is absent. Here $\epsilon$ is a normalized detuning parameter given by $\Omega_{\max } / \Omega$. The left spot is due to diffraction in the fundamental grating; the right is due to the directly transmitted readout beam. 


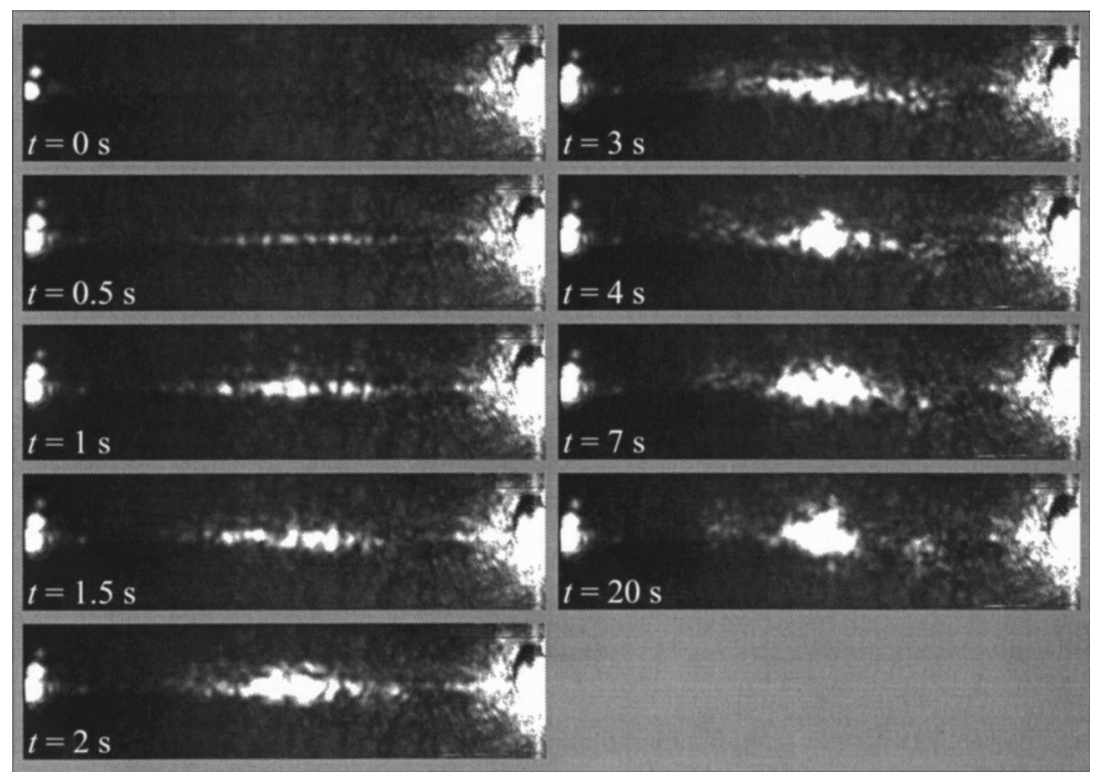

Fig. 5. Transient evolution of the diffraction pattern at $\epsilon=0.20$.

angle. The result is shown in Fig. 4, where each picture represents steady state. For $0.165 \leqslant \epsilon \leqslant 0.21$ we observed, apart from the fundamental and zeroth-order diffraction spots, effectively three spots: one centered at $\mathbf{K} / 2$ and two others whose centers tended to move from approximately $(\mathbf{K} / 4,3 \mathbf{K} / 4)$ to $(\mathbf{K} / 3,2 \mathbf{K} / 3)$ as $\epsilon$ was increased. Since the positions of these spots change continuously, we call them simply side spots to the $\mathbf{K} / 2$ spot, rather than subharmonics. The gratings are correspondingly called side gratings. For $\epsilon \geq 0.21$ only the $\mathbf{K} / 2$ spot was present. As regards the strengths of the spots the $\mathbf{K} / 2$ spot showed maximum strength at $\epsilon \simeq 0.25$, whereas the two side spots seemed to have maximum strengths at $\epsilon \simeq 0.18$.

Also worth noting in Fig. 4 is that both the $\mathbf{K} / 2$ and the side spots are generally much broader than the first-order spot; this indicates that these spots cannot be due to single gratings but rather to packages of gratings. (Note, however, that the broad appearance of the first-order spot for large $\epsilon$ is simply due to the increasing strength of the first-order diffraction as $\epsilon$ approaches 1 . Then, of course, the intensity of the scattered light around the first-order beam increases, too, leading to the broad spot).

Finally, it should be mentioned that by varying $E_{0}$ from 6 to $15 \mathrm{kV} \mathrm{cm}^{-1}$, we found that the side spots were most pronounced for $E_{0}$ around $8-9 \mathrm{kV} \mathrm{cm}^{-1}$. This is quite surprising, because usually all PPO effects increase in strength with $E_{0}$.

\section{B. Transient Case}

As mentioned above, the images in Fig. 4 represent steady state. But how do they develop to get to that state? Does each spot simply increase in strength until steady state is reached, or do the patterns change shape transiently before reaching the shapes in Fig. 4? To elucidate these interesting questions, we chose the case $\epsilon$ $=0.20$. We then provisionally switched $\Sigma$ in Fig. 1 to position 0 so that the piezo mirror performed a rapid sine movement. This significantly reduces the strength of the fundamental grating so that the PPO process fell below threshold. The technique is well known for switching gratings on and off. ${ }^{23,24} \Sigma$ was then reset to position 1 , and the series of images shown in Fig. 5 was recorded in the transient period of growth of the PPO process. At the beginning of the period one can see that a large continuum of gratings, all apparently with grating vectors parallel to $\mathbf{K}$, are excited. The outermost gratings, though, seem to stop their growth rather early; thus the central gratings become dominant. In the end, though, the side spots survive, so the final result consists of three spots as in Fig. 4 for $\epsilon=0.20$. For comparison we measured the rise time of the fundamental spot, which turned out to be $\sim 100 \mathrm{~ms}$. Hence the development of the PPO pattern is quite slow in comparison with the rise time of the fundamental grating.

\section{DISCUSSION}

By comparing the two sets of pictures in Figs. 2 and 4, the influence of beam coupling on the PPO process can be judged. The first thing to note is that the transversal split of the $\mathbf{K} / 2$ spot, observed in the traditional configuration, is not observed in the new geometry. Hence, the transversal split seems to be due to beam coupling. Even when the applied electric field was increased there was no sign of transversal split in the new configuration. Apart from this, the existence, as well as the order of appearance, of the $\mathbf{K} / 2$ spot and its side spots seems to be the same for the two configurations. The appearance itself of the side spots in the new geometry may be found surprising-partly because they have not been observed before and partly because of the recently published results on ac subharmonics, ${ }^{17}$ in which no such division of gratings into groups of subharmonics could be obtained. 
Is the term subharmonic generation then appropriate to use in the dc case? Or, in other words, would it be reasonable to say that the diffraction patterns in Fig. 4 are due to distinct gratings, with grating vectors assuming integer fractions of $\mathbf{K}$ ? Evidently not. There are two reasons: (i) the PPO diffraction spots are so broad that a continuum of gratings must be present, and (ii) the longitudinal splitting between the side spots seems to shift continuously with $\Omega$.

As yet, the theoretical models of PPO have not been capable of describing continua of gratings, so the theoretical description of the results in Fig. 4 may take some time. The diffraction spots observed for $\epsilon=0.165$, however, seem quite confined, so there might be a chance that the PPO process for low values of $\epsilon$ can be described by the present theory. The recent theoretical prediction ${ }^{18}$ of a modulationally unstable $\mathbf{K} / 2$ grating around $\epsilon=0.25$ is virtually confirmed by the broad spot in Fig. 4 at $\epsilon$ $=0.24$, in the sense that the broadening must be due to a continuum of gratings around $\mathbf{K} / 2$.

The transient evolution in Fig. 5 reveals important news as well. First, it is quite surprising that at the beginning of the growth, i.e., at $t=0.5 \mathrm{~s}$, all PPO grating vectors seem to be aligned along $\mathbf{K}$. According to the theory ${ }^{2}$ there should be a weak dependence on the transversal component of the PPO grating vectors. The rather well-confined line of diffracted light in Fig. $5, t=0.5 \mathrm{~s}$, points to a much sharper dependence. The remaining images illustrate that the growth is stabilized by an isotropic broadening (i.e., equal broadening in all directions) of the $\mathbf{K} / 2$ grating and by excitation of two broad side gratings.

The present findings are very useful for both theorists and experimentalists in the field of photorefractive parametric oscillation (PPO). As mentioned, the vast majority of experiments on subharmonics and PPO have been performed in traditional geometry, which involves beam coupling. And, paradoxically, the vast majority of theoretical models that try to describe the results of these experiments do not include beam coupling. As beam coupling has proven to have a significant influence in the traditional configuration, it seems obvious that new experiments performed in the new geometry are needed to characterize PPO in detail, so that the present theoretical models can be properly verified. The purpose of the present paper is, however, only to prove the influence of beam coupling, not to perform this detailed analysis.

\section{ACKNOWLEDGMENTS}

H. C. Pedersen and P. M. Johansen were supported by the Danish Natural Science Research Council, grants 9502764 and 9600986. Financial support of the International Association for the Promotion of Cooperation with Scientists project 96-0954 is also gratefully acknowledged.

\section{REFERENCES}

1. S. Mallick, B. Imbert, H. Ducollet, J.-P. Herriau, and J. P. Huignard, "Generation of spatial subharmonics by two- wave mixing in a nonlinear photorefractive medium," J. Appl. Phys. 63, 5660-5663 (1988).

2. B. I. Sturman, M. Mann, J. Otten, and K. H. Ringhofer, "Space-charge waves in photorefractive crystals and their parametric excitation,” J. Opt. Soc. Am. B 10, 1919-1932 (1993).

3. See, for example, J.-P. Huignard and A. Marrakchi, "Coherent signal beam amplification in two-wave mixing experiments with photorefractive $\mathrm{Bi}_{12} \mathrm{SiO}_{20}$ crystals," Opt. Commun. 38, 249-254 (1981); Ph. Réfrégier, L. Solymar, H. Rajbenbach, and J.-P. Huignard, "Two-beam coupling in photorefractive $\mathrm{Bi}_{12} \mathrm{SiO}_{20}$ crystals with moving grating: theory and experiments," J. Appl. Phys. 58, 45-57 (1985).

4. H. C. Pedersen and P. M. Johansen, "Parametric oscillation in photorefractive media," J. Opt. Soc. Am. B 12, 10651073 (1995).

5. J. Takacs and L. Solymar, "Observation of split subharmonics in a $\mathrm{Bi}_{12} \mathrm{SiO}_{20}$ crystal" (personal communication, 1991).

6. H. C. Pedersen and P. M. Johansen, "Observation of angularly tilted subharmonic gratings in photorefractive bismuth silicon oxide," Opt. Lett. 19, 1418-1420 (1994).

7. H. C. Pedersen and P. M. Johansen, "Longitudinal, degenerate, and transversal parametric oscillation in photorefractive media," Phys. Rev. Lett. 77, 3106-3109 (1996).

8. H. C. Pedersen and P. M. Johansen, "Longitudinal, degenerate, and transversal photorefractive parametric oscillation: theory and experiment," J. Opt. Soc. Am. B 14, 1418-1427 (1997)

9. C. H. Kwak, M. Shamonin, J. Takacs, and L. Solymar, "Spatial subharmonics in photorefractive $\mathrm{Bi}_{12} \mathrm{SiO}_{20}$ crystal with a square wave applied field," Appl. Phys. Lett. 62, 328-330 (1993).

10. T. E. McClelland, D. J. Webb, B. I. Sturman, and K. H. Ringhofer, "Generation of spatial subharmonic gratings in the absence of photorefractive beam coupling," Phys. Rev. Lett. 73, 3082-3084 (1994).

11. L. B. Au, L. Solymar, and K. H. Ringhofer, "Subharmonics in BSO," in Technical Digest on Photorefractive Materials, Effects and Devices II (Société Francais d'Optique, Aussois, France, 1990), pp. 87-91.

12. O. P. Nestiorkin, "Instability of spatial subharmonics under hologram recording in a photorefractive crystal," Opt. Commun. 81, 315-320 (1991).

13. B. I. Sturman, A. Bledowski, J. Otten, and K. H. Ringhofer, "Spatial subharmonics in photorefractive crystals," J. Opt. Soc. Am. B 9, 672-681 (1992).

14. D. J. Webb and L. Solymar, "Observation of spatial subharmonics arising during two-wave mixing in BSO," Opt. Commun. 74, 386-388 (1990).

15. D. J. Webb, L. B. Au, D. C. Jones, and L. Solymar, "Onset of subharmonics generated by forward interactions in $\mathrm{Bi}_{12} \mathrm{SiO}_{20}$," Appl. Phys. Lett. 57, 1602-1604 (1990).

16. J. Takacs, M. Schaub, and L. Solymar, "Subharmonics in photorefractive $\mathrm{Bi}_{12} \mathrm{TiO}_{20}$ crystals," Opt. Commun. 91, 252254 (1992).

17. H. C. Pedersen, P. M. Johansen, and D. J. Webb, "Photorefractive subharmonics-a beam coupling effect?" J. Opt. Soc. Am. B 15, 1528-1532 (1998).

18. B. I. Sturman, M. Aguilar, F. Agullo-Lopez, and K. H. Ringhofer, "Fundamentals of the nonlinear theory of photorefractive subharmonics," Phys. Rev. E 55, 6072 (1997).

19. E. V. Podivilov, H. C. Pedersen, P. M. Johansen, and B. I. Sturman, "Transversal parametric oscillation and it's external stability in photorefractive sillenite crystals," Phys. Rev. E 57, 6112-6126 (1998).

20. See, for example, A. Marrakchi, R. V. Johnson, and A. R. Tanguay, Jr., "Polarization properties of photorefractive diffraction in electrooptic and optically active sillenite crystals (Bragg regime)," J. Opt. Soc. Am. B 3, 321-336 (1986); or P. Yeh, Introduction to Photorefractive Nonlinear Optics (Wiley, New York, 1993).

21. T. E. McClelland, D. J. Webb, B. I. Sturman, M. Mann, and K. H. Ringhofer, "Low frequency peculiarities of the photo- 
refractive response in sillenites," Opt. Commun. 113, 371377 (1995).

22. T. E. McClelland, D. J. Webb, B. I. Sturman, E. Shamonina, M. Mann, and K. H. Ringhofer, "Excitation of higher spatial harmonics by a moving light pattern in sillenites," Opt. Commun. 131, 315-321 (1996).

23. P. M. Johansen, "Enhanced four-wave mixing in photore- fractive BSO produced by temporal phase shifts," J. Phys. D 22, 247-253 (1989).

24. P. E. Andersen, P. Buchhave, P. M. Petersen, and M. V. Vasnetsov, "Nonlinear combinations of gratings in $\mathrm{Bi}_{12} \mathrm{SiO}_{20}$ : theory and experiment," J. Opt. Soc. Am. B 12, 1422-1433 (1995). 